\title{
Die deutsch-unitarische Entwicklung seit 1945
}

\author{
Jörg Last
}

In Dan McKanans Beitrag ist von „Accidents in Unitarian History“ die Rede; „Accidents“ lässt sich mit „zufällige Begebenheiten“ übersetzen, aber auch mit „Unfälle“. Dan McKanans Einladung, diese „Unfälle“ unitarischer Geschichte in Deutschland zu reflektieren, wollen wir deutschen Unitarier gerne annehmen.

Mein Beitrag zur deutsch-unitarischen Entwicklung seit 1945 will nur ein bescheidener Anstoß sein, diese wichtige Aufgabe - gerne im Weiteren auch gemeinsam mit anderen unitarischen und freireligiösen Gruppen in Deutschland - anzugehen. Ich bin überzeugt, wir können alle dadurch nur gewinnen - und damit auch die unitarische Idee!

\section{Die freiprotestantischen Ursprünge}

Die Unitarier in Deutschland stehen maßgeblich in der Tradition der rheinhessischen Freiprotestanten, deren Gemeinschaft 1876 gegründet und 1902 als Verein eingetragen wurde. Sie waren zu dieser Zeit eine regional begrenzte, freichristliche Laienvereinigung von fünfzehn Gemeinden mit nicht ganz tausend Mitgliedern. Ihre Leitung und die Mitglieder der Gemeinschaftsgremien wurden von einer Generalversammlung auf fünf Jahre gewählt. ${ }^{1}$

\footnotetext{
${ }^{1}$ Friedrich Ehrlicher: Deutsche Unitarier - Deutsches Kulturwerk: Eine Beqiebung in Spannungen. München 1988 (gekürzt veröffentlicht in MdP 2/1988 der Deutschen Unitarier Religionsgemeinschaft e.V.), S. 2.
} 
Rheinhessen gehörte seit 1816 zum Großherzogtum Hessen und war zuvor zwanzig Jahre lang Teil des revolutionären Frankreich. Die damals gewonnenen politischen und gesellschaftlichen Freiheiten formten die Identität der Menschen in der überwiegend landwirtschaftlich geprägten Region, so dass Rheinhessen auch ein Zentrum der Märzrevolution 1848 war. Die Menschen suchten die geistigreligiöse Freiheit und zweifelten zunehmend an den kirchlichen Lehren. Als die Landeskirchen 1875 die Kirchensteuer einführten, war der Unmut so groß, dass viele die Kirchen verließen, um eigene, „freie“ Gemeinschaften zu bilden. Das Glaubensbekenntnis der freien Protestanten war kurz und einfach:

Wir glauben an Gott, den allmächtigen Geist im Weltall. Wir glauben an Jesus Christus als den begeistertsten und den begabtesten Lehrer der Menschen, der sein Leben geopfert hat für seine Lehre, der ein Erlöser ist aller derer, welche seine Lehre nicht nur glauben, sondern sie beherzigen und in ihrem Leben befolgen. Wir glauben an einen heiligen Geist, wirkend als sittlicher Gesamtgeist der Menschen, der sie in fortschreitender Entwicklung zu edler Menschlichkeit, Bildung und Sitte führt. Wir glauben an das Reich Gottes, als das Reich der Wahrheit, Gerechtigkeit und Bruderliebe, und halten es für die Aufgabe der christlichen Kirche, dieses Reich mehr und mehr $\mathrm{zu}$ verwirklichen. Wir glauben an ein ewiges Leben, denn wir sehen um uns her keine Vernichtung, sondern nur Wechsel der äußeren Erscheinungen. ${ }^{2}$

Seit 1909 war der ehemalige evangelische Pfarrer Rudolf Walbaum als ihr „Geistlicher Leiter" angestellt. Auf dem Weltkongress für Freies Christentum und Religiösen Fortschritt 1910 in Berlin ${ }^{3}$ kam er in Kontakt mit englischen und amerikanischen Unitariern und erkannte das verwandte Denken mit dem seiner Gemeinschaft. Deren Zeitschrift Der Freiprotestant bekam bald darauf den Untertitel Deutsch-unitarische Blätter. 1934 öffnete sich die Gemeinschaft für Diaspora-Mitglieder auch außerhalb Rheinhessens, und Walbaum begann, weiträumige Kontakte zu kirchenkritischen Kreisen in Deutschland zu pflegen. ${ }^{4}$

Damit ist ein historischer Startpunkt der Entwicklung beschrieben, die zu den heutigen deutschen Unitariern führte. Die dazwischen liegende Zeit war nicht ohne Spannungen und Krisen und böte mit Sicherheit Stoff für eine ganze Reihe von eigenen Tagungen. Ich beschränke mich hier auf eine kurze Beschreibung der heutigen Gemeinschaft, um dann an ein paar Beispielen die historische Entwicklung aufzuzeigen.

2 Christian Elßner: Materialien zum Religionsunterricht und zur Selbstbelehrung für Schule und Haus. Alzey 1882, S. 92.

3 Vgl. dazu den Beitrag von Sturm-Berger in diesem Band.

4 Ehrlicher: Deutsche Unitarier, S. 2. 


\section{Die Unitarier - Religionsgemeinschaft freien Glaubens}

Die Unitarier-Religionsgemeinschaft freien Glaubens hat heute rund 700 Mitglieder im gesamten Bundesgebiet. Gemeinden, Gruppen und Einzelmitglieder sind jeweils zu Landesgemeinden zusammengefasst, die wiederum Mitglied in der Gesamtgemeinschaft sind. Die Gruppierungen verwalten sich selbst und entsenden Delegierte zu der alle zwei Jahre tagenden Hauptversammlung. Dort werden das Präsidium und die Gremien jeweils für vier Jahre gewählt.

Ein besonderes Gremium ist der Geistige Rat. Er besteht aus bis zu neun Mitgliedern und berät den Vorstand. Seine Aufgabe ist es, die geistig-religiösen Gemeinsamkeiten der Mitglieder bewusst zu machen und zu formulieren. Dazu nimmt er die Anregungen der Mitglieder auf und gibt Impulse für die Weiterentwicklung in die Gemeinschaft zurück. Er äußert sich zu Fragen, die aus der Gemeinschaft an ihn herangetragen werden, arbeitet aber auch auf eigene Initiative. ${ }^{5}$

Wir Deutschen Unitarier haben - anders als noch die Freien Protestanten kein Bekenntnis. Wir haben unsere verbindenden religiösen Vorstellungen in den sogenannten „Grundgedanken“ formuliert. Darin finden sich Aussagen zu unserem Religionsverständnis, zum unitarischen Glauben, dem Leben im Allgemeinen, zu uns Menschen im Besonderen und zu unserem Zusammenleben. Die Aussagen wurden in einem demokratischen Prozess entwickelt und in der aktuellen Fassung auf der Hauptversammlung 1995 einstimmig beschlossen.

Jugend-, Familien- und Seniorenbetreuung haben einen festen Platz in den Aktivitäten der Gemeinschaft. Die Gruppen und Gemeinden veranstalten Feste im Jahreskreis sowie Feierstunden zu verschiedenen Themen. Als reine Laiengemeinschaft organisieren die Mitglieder alle Feiern und Feste selbst und führen die Lebens-, Jugend- und Eheleiten sowie Trauerfeiern nach eigenen Vorstellungen durch. Vorgegebene Formen oder feste Rituale existieren nicht. Es gilt das Motto: „Jeder darf, nichts muss, alles kann!“

Seit 1959 treffen wir uns alle zwei Jahre zum Unitariertag, einer großen öffentlichen Veranstaltung. Daran nehmen auch Freunde und Gäste aus aller Welt teil. Ich könnte jetzt noch von den angeschlossenen Organisationen - einer Akademie, einem Jugendbund, einem Hilfswerk oder einem Jugend- und Familienbildungswerk - berichten, will aber abschließend nur noch darauf hinweisen, dass die deutschen Unitarier auch noch Mitglied im Dachverband freier Weltanschanungsgemeinschaften (DFW), dem Internationalen Rat der Unitarier und Universalisten (ICUU) und der Internationalen Vereinigung für religiösen Frieden (IARF) sind.

Und damit zurück ins Jahr 1945.

\footnotetext{
${ }^{5}$ Deutsche Unitarier Religionsgemeinschaft e.V.: Verfassung. Hitzacker 1995.
} 


\section{Die Entwicklung seit 1945}

Nach dem Kriegsende bot der achtundsiebzigjährige Rudolf Walbaum „religiös Heimatlosen“ die Mitgliedschaft in seiner freiprotestantischen Gemeinschaft an. Schon bald gründeten sich neue Gruppen und Gemeinden in Norddeutschland, wodurch die Gemeinschaft schnell wuchs. ${ }^{6}$ Zudem engagierten sich die Freiprotestanten auch in der Seelsorge in den alliierten Internierungslagern.

\section{Herbert Böhme und Eberhard Achterberg}

1947 wurde so Herbert Böhme im Lager Hohenasperg auf die Gemeinschaft aufmerksam. ${ }^{7}$ Böhme war im Dritten Reich eine prominente Figur; er war NSDAPMitglied, SA-Obersturmführer, Fachschaftsleiter der Reichsschrifttumskammer und nicht zuletzt ein bekannter Dichter. Er gründete noch vor seiner Entlassung aus der Internierungshaft im Lager eine neue Gruppe der Religionsgemeinschaft und nahm im Herbst an einem Treffen auf dem Klüt, einem Berg nahe Hameln, teil. Walbaum hatte dorthin die führenden Personen der gewachsenen Freiprotestanten zu Planungsgesprächen eingeladen. ${ }^{8}$

Walbaum formuliert 1946 in seinem sechzehnseitigen Heft Religiöser Unitarismus folgende Hauptprinzipien, die eine unitarische Gemeinschaft charakterisieren:

1. Vollständige geistige Freiheit in religiöser Hinsicht statt Gebundensein an Glaubensbekenntnisse oder Konfessionen. 2. Uneingeschränkter Gebrauch der Vernunft in Dingen der Religion statt Verlass auf äußere Autorität oder Tradition der Vergangenheit. 3. Weitgehende Toleranz gegenüber den verschiedenen religiösen Ansichten und Bräuchen statt Beharren auf Gleichförmigkeit in Lehre, Gottesdienst oder Verfassung. ${ }^{9}$

Damit stellte er sich ganz bewusst in die von Earl Morse Wilbur dokumentierte Tradition der antitrinitarischen Bewegung, die im 4. Jahrhundert ihren Anfang nahm und sich zwölf Jahrhunderte später in Siebenbürgen und Polen fortsetzte. „Deutsch-unitarisch“ sei darüber hinaus, schrieb Walbaum, „eine Weltanschauung, die Gott und Welt nicht dualistisch ganz voneinander trennt, aber auch nicht monistisch vereinerleit, sondern zu einer All-Einheit verbunden sieht, nach welcher auf Erden der Mensch als solcher der eingeborene Gottessohn ist. "10

In der Gemeinschaft bildete sich durch die vielen Neuzugänge aber ein spannungsreiches Spektrum religiös-weltanschaulicher Ansichten, das von den linksrheinischen Freiprotestanten über eine liberale Gruppierung ehemaliger Deutsch-

\footnotetext{
${ }^{6}$ Ehrlicher: Deutsche Unitarier, S. 2f.

${ }^{7}$ Ulrich Nanko: Religiöse Gruppenbildungen vormaliger „Deutschgläubiger" nach 1945. In: Antisemitismus, Paganismus, Völkische Religion. Hg. von Hubert Cancik und Uwe Puschner. München 2004, S. 121-134.

${ }^{8}$ Ehrlicher: Deutsche Unitarier, S. 3 f.

${ }^{9}$ Rudolf Walbaum: Religiöser Unitarismus. Stuttgart 1946, S. 4.

${ }^{10}$ Ebd.
} 
gläubiger bis hin zu antiklerikalen, antichristlichen und auch antisemitischen Personen reichte. ${ }^{11}$ Hans-Dietrich Kahl formuliert:

wir erlebten den Anschluss neuer Gemeinschaften in Schleswig-Holstein und Nordhessen, die unabhängig aus eigenen Wurzeln entstanden, die [ihre] Gemeinsamkeit auf gegenwärtige Erfahrungen gründeten, nicht auf Wachstum von früher her. Sie hatten wenig innere Fühlung mit der älteren [unitarischen] Tradition [...] [und] stellten im Rahmen des Ganzen alsbald einen bedeutenden Anteil der Mitgliederzahl. ${ }^{12}$

Die bis dahin neugegründeten zwölf rechtsrheinischen Gemeinden umfassten insgesamt 800 Mitglieder. In den sogenannten „Urgemeinden“ links des Rheins waren es zu dieser Zeit mit 950 Mitgliedern nur wenige mehr.

Auf dem ersten Klüt-Treffen beeindruckte der junge Eberhard Achterberg mit einem Vortrag zum Thema Deutschlands Zukunft als religiöse Aufgabe. ${ }^{13}$ Achterberg war neunzehnjährig der NSDAP beigetreten und hatte dem Dritten Reich überzeugt und aktiv gedient. ${ }^{14}$ In seinem Vortrag auf dem Klüt vollzog Achterberg aber eine ,scharfe Abrechnung mit dem unheilvollen ,Führerprinzip“ der zurückliegenden Jahre“ und appellierte an die ,unaufhebbare persönlich-sittliche Verantwortlichkeit des einzelnen“. Seine Ausführungen hinterließen einen tiefen Eindruck bei den Teilnehmern. ${ }^{15}$ „Weil ich damals aktiv und überzeugt dabei war, deshalb trete ich dafür ein, dass sich eine solche Entwicklung nicht wiederholen darf“, schrieb Achterberg 1983 in einem Brief an den jüdischen Schriftsteller Erich Fried. ${ }^{16}$

\section{Der Klütkreis}

In Herbert Böhme reifte derweil der Plan, die anwesende Gruppe zur elitären Führungsspitze der Freien Protestanten unter seiner Leitung zu machen, und er bezeichnete sie fortan als Klütkreis; ${ }^{17}$ ein Gremium, das bis dahin in der Satzung des Vereins der Freiprotestanten nicht vorgesehen war. Ab Januar 1948 gab Böhme zudem im Namen des Klütkreises ein eigenes Nachrichtenblatt namens Klüter Blätter heraus. Böhme rechnete wohl mittlerweile auch fest damit, auf dem nächsten Treffen von Walbaum als dessen Nachfolger in der Funktion des „Geistlichen

\footnotetext{
11 Vgl. Nanko: Religiöse Gruppenbildungen.

12 Hans-Dietrich Kahl: 1996 - ein Jahr des Erinnerns. In: unitarische blätter. Bd. 1. Jan./Feb. 1996, S. 3839, hier S. 39.

13 Vgl. Nanko: Religiöse Gruppenbildungen.

14 Achterberg diente zunächst als stellvertretender Schriftleiter, dann als Hauptschriftleiter der Nationalsozialistischen Monatshefte in der Dienststelle des Reichsleiters Rosenberg.

${ }_{15}$ Hans-Dietrich Kahl: Strömungen. Die Deutschen Unitarier seit 1945 - ein kritischer Rückblick. In: unitarische befte. Nr. 4. München 1989.

${ }_{16}$ Hans-Dietrich Kahl: Eberhard Achterberg. In: unitarische blätter. Bd. 2. März/April 2010, S. 91-97, hier S. 96 .

17 Vgl. Ehrlicher: Deutsche Unitarier.
} 
Leiters“ der Freiprotestanten vorgeschlagen zu werden. Am 6. April 1948 verstarb Walbaum jedoch kurz vor der Tagung des Klütkreises.

An dieser Tagung nahm der amtierende Vorsitzende des eingetragenen Vereins nur zeitweise teil, die Ergebnisse des Treffens waren dennoch weitreichend: Es wurden Richtlinien für die Gemeindegründung verabschiedet, die Zusammensetzung des „Führungskreises“ und von zwölf Arbeitskreisen bestimmt und die Wahl von Sprechern der Gemeinschaft vorgenommen. Herbert Böhme wurde als „1. Sprecher" gewählt und auch der Leiter des Führungskreises. ${ }^{18}$

\section{Die Eppelsheimer Formel}

Auf einer außerordentlichen Generalversammlung des Vereins Freier Protestanten im September in Eppelsheim wurden die Beschlüsse des Klüt-Treffens aber nur zum kleinen Teil anerkannt. Böhme wurde als „1. Sprecher“ nicht bestätigt, und der Klütkreis wurde dem Ältestenrat, einem neugegründeten Führungsgremium der Landesgemeindeleiter, unterstellt. Die Generalversammlung beschloss zudem die sogenannte Eppelsheimer Formel. Sie lautet:

Die Religionsgemeinschaft Freier Protestanten setzt sich eindeutig ab vom dogmatischen Kirchenchristentum, aber auch von allem Antichristentum wie von jeder feindlichen Frontstellung gegen andere religiöse Auffassungen überhaupt. Sie sucht das Erbe unserer bisherigen christlich-abendländischen Glaubensgeschichte für die religiöse Weiterentwicklung fruchtbar zu machen, lehnt es aber ab, sich an dieses Erbe zu binden. Als vorläufige Richtschnur gelten ihr die auf Seite 13 bis 14 der Schrift Religiöser Unitarismus von Rudolf Walbaum aufgestellten 10 Punkte.

Diese zehn Punkte lassen sich durch

folgende Stichworte kennzeichnen: Weltanschauung der All-Einheit, panentheistische Vorstellung eines unpersönlichen Gottes, undogmatisches religiöses Denken mit weiter Toleranz, eine liberale, soziale und national eingestellte friedliche Ethik, eine Atmosphäre warmherziger, offener Humanität. ${ }^{19}$

Böhme war enttäuscht, dass er seine Pläne zur Gestaltung der Gemeinschaft nicht gegen die Mehrheit der „Urgemeinden“ durchsetzen konnte. Besonders aber störte ihn, dass der Anspruch des Klütkreises auf die zentrale geistige Führung der Gemeinschaft nicht akzeptiert wurde und dieser nur eine beratende Stimme haben

\footnotetext{
18 Ebd., S. 4

${ }^{19}$ Hans-Dietrich Kahl: Die Eppelsheimer Formel. Glaube und Tat. Hamburg 1949.
} 
sollte. Dabei berief er sich auf einen Führungsauftrag, den er von Walbaum erhalten haben wollte.

Auf höchste erzürnt soll er über die im Februar erscheinende Ausgabe des Mitteilungsblattes der Gemeinschaft Glaube und Tat gewesen sein. In diesem wurde über die Eppelsheimer Formel berichtet, ohne jedoch auf ihn als Person oder auf seine nationalen Vorstellungen einzugehen. Böhme sah in der Gemeinschaft „,kein[en] Bürgerverein, sondern die Gestaltwerdung eines in uns allen lebendigen Kulturwillens“. In zwei Rundschreiben rief Böhme im März die mit ihm sympathisierenden Gemeinden zur „Lossage von Alzey“ und der Eppelsheimer Formel auf. Er wollte mit ihnen einen neuen Verein unter eigenen Namen gründen. ${ }^{20}$

Offenbar verfolgte er seine völkisch-nationalen Ziele mit aller Konsequenz. Die liberale und tolerante Gegenseite tat sich schwer mit einer angemessenen Antwort, wollte sie doch die Gemeinschaft nicht spalten. Aus dem Respekt vor der eigenständigen Entwicklung jedes einzelnen setzte sie lange auf das Prinzip des Dialogs. In ihrer progressiven Haltung waren die Deutschen Unitarier dem Zeitgeist voraus, antiautoritäre Prinzipien als Antwort auf die Erfahrungen im Nationalsozialismus standen ihnen in dieser Situation aber im Weg.

Eberhard Achterberg beschäftigten besonders Fragen der Wertorientierung, der antiautoritären Erziehung, der Gesellschaftspolitik und des persönlichen Miteinanders. 1975 resümiert er den Konflikt in einer Rückschau auf seine langjährige Tätigkeit als Schriftleiter von Glaube und Tat.

Sollte der Weg in die Zukunft zu einer Fortsetzung dessen führen, was in der Glaubensbewegung aufgebrochen war? Deutscher Glaube als Ausdruck deutscher Wesensart und darum deutsche Unitarier? - Deutsches Brauchtum in Fest und Feier als Bewahrung deutschen Kulturerbes? - Oder sollte der Weg in die Zukunft als kühne Pioniertat in ein neues Land des Glaubens vorstoßen? Eine ganz neue Art von Religion, die ihren Wurzelgrund im Menschlichen hat und auch in Fest und Feier nach neuer Gestaltung, neuer Sinngebung sucht und darum vom Unitariertum spricht als einer weltweiten Bewegung. Unitariertum als Verkündung eines neuen Humanismus und darum auch als Wegbereiter einer neuen Ethik? ${ }^{21}$

Hier deutet er schon an, dass die implizite Politisierung der Gemeinschaft durch Böhme zunehmend Widerstand hervorrief. Kaum jemand suchte jedoch die direkte Auseinandersetzung mit ihm. In der demokratisch verfassten Gemeinschaft war man - geprägt vom gegenseitigen Respekt - gewohnt, mit einander um Wahrheiten zu ringen und zu streiten. Als „Suchender“ begegnete der selbstverantwortliche Mensch anderen Meinungen dabei tolerant, undogmatisch und offen. Doch einer

${ }^{20}$ Ehrlicher: Deutsche Unitarier, S. 6.

${ }^{21}$ Eberhard Achterberg: Was ich gewollt habe. Glaube und Tat. In: Deutsch-unitarische Blätter. Bd. 1, Januar 1975, S. 6-8, hier S. 7. 
Person von solcher Überzeugung und mit diesem Führungsanspruch und Sendungsbewusstsein hatte die Gemeinschaft anfangs wenig entgegenzusetzen.

„Zwei extreme Strömungen zeichneten sich u.a. schon sehr bald ab, und wenn sich auch mitunter verschiedene Standpunkte zu Gegensätzen verschärften, so gelang es doch immer wieder, Brücken des Gemeinsamen zu schlagen", schreibt Achterberg weiter,

ich wollte nichts zerstören, ich wollte nicht verletzen, und ich wollte, dass beide Strömungen innerhalb der großen Gemeinschaft ihre Heimat finden. Aber was den Weg in die Zukunft betrifft, so konnte der nach meiner Meinung nur in der einen Richtung weitergehen. In der Richtung eines weltweiten Unitariertums, in der Richtung des Abbaus von Rassenvorurteilen und einer wachsenden Völkerverständigung. In der Richtung einer neuen Religion ohne Gott, in einer Richtung einer Ethik der Mitmenschlichkeit. ${ }^{22}$

In dieser „Richtung“ wurde dann beispielsweise auch an den ersten religiösen Leitsätzen gearbeitet, um ein gemeinschaftliches Fundament für die individuelle Entwicklung des Einzelnen zu schaffen.

\section{Die Spaltung zeichnet sich ab}

Abwechselnd tagten in dieser Zeit die Versammlungen auf dem Klüt und in Eppelsheim. Was die Gruppe um Böhme dort beschloss, wurde hier vom offiziellen Verein nicht oder nur teilweise mitgetragen. Als in Eppelsheim Bedingungen für eine weitere Zusammengehörigkeit mit den rechtsrheinischen Gemeinden gestellt wurden, beschloss der auf dem Klüt gewählte Gemeinschaftsrat die Trennung von den „Urgemeinden“. Diese lenkten daraufhin ein. Schließlich einigte man sich auf eine neue außerordentliche Generalversammlung im kommenden Jahr.

Auf dieser Sitzung im Februar 1950 wurde der neue Name Deutsche Unitarier, Religionsgemeinschaft offiziell gebilligt, eine neue Verfassung beschlossen und die Gremien neu geordnet. Böhme wurde als „Leiter des neu zu bildenden Klütkreises" Mitglied im Gemeinschaftsrat. Trotzdem war er nicht zufrieden. Mit der Gründung des Deutschen Kulturwerks europäischen Geistes schaffte sich Böhme ein Wirkungsfeld, das unabhängig von der Religionsgemeinschaft war, und das er ganz nach seinen Vorstellungen gestalten konnte.

Doch von der Gemeinschaft wollte er nicht lassen, obwohl ihm nun verstärkt Widerstand entgegengebracht wurde, auch in der Öffentlichkeit. Böhme erregte wegen neonazistischer Äußerungen und Veröffentlichungen Anstoß. Diese fielen auf die Gemeinschaft zurück, obwohl die Hauptversammlung zuvor einstimmig beschlossen hatte, dass die Religionsgemeinschaft als solche jede parteipolitische Stellungnahme ablehnt - ein stumpfes Schwert.

\footnotetext{
22 Ebd.
} 
Die Auseinandersetzung innerhalb der Gemeinschaft eskalierte langsam und Böhme geriet mehr und mehr in die Defensive. Anfang 1953 schrieb er in seinem Rundbrief: „Es ist nicht mein Ehrgeiz, als Erster Sprecher gewählt, sondern als Euer Erster Sprecher gewürdigt zu sein. Wo Ihr mir diese Würdigung absprecht, bitte ich, mich verabschieden zu dürfen." Doch es brauchte noch weitere Monate bis er sich tatsächlich zurückzog. Insgesamt sieben Jahre waren seit dem ersten Klüt-Treffen vergangen - eine lange Zeit. Die Bilanz danach: Einige Gemeinden insbesondere aus dem Rheinhessischen - hatten sich von der Gemeinschaft getrennt.

\section{Leitgedanken}

1957 wurden die Leitgedanken auf der Hauptversammlung in Hameln einstimmig angenommen. Damit waren erstmals religiöse Glaubensaussagen gemeinschaftlich erarbeitet und demokratisch beschlossen worden. Sie hatten jedoch keinen Bekenntnischarakter und waren veränderbar - eine religionsgeschichtliche Neuheit!23

Die Causa Böhme sollte aber nicht die einzige Prüfung in dieser Hinsicht bleiben. Eine Religionsgemeinschaft, die die Vielfalt der Vorstellungen und Meinungen ihrer Mitglieder als einen hohen Wert versteht, aus denen die Gemeinschaft schöpfen und sich weiterentwickeln kann, ist aufgrund dieses Idealismus verletzlich, denn sie besitzt kein Immunsystem, das Ideen oder Meinungen sofort als falsch erkennen und abwehren kann.

\section{Sigrid Hunke}

1969 hielt die Religionswissenschaftlerin und Schriftstellerin Sigrid Hunke auf dem Unitariertag einen Gastvortrag über Die andere Religion Europas. Bald danach wurde sie Mitglied. Anknüpfungspunkte ihrer neuheidnischen Ideenwelt zu den Gedanken Walbaums von der „Einheit des Allseins“ waren wohl gegeben, auch wenn es andere Differenzen gab. Ihr Wissen und ihr Auftreten verschafften ihr Respekt, doch nur wenige wussten zu dieser Zeit wohl von ihrem politischen und wissenschaftlichen Wirken im Dritten Reich. ${ }^{24}$

Hunke wurde 1971 zur Vizepräsidentin der Gemeinschaft gewählt. Sie schien den Mitgliedern eine gute Kompromisskandidatin zwischen den nach wie vor bestehenden Flügeln innerhalb der Deutschen Unitarier zu sein, doch sie wird die Religionsgemeinschaft letztlich spalten. Hunke zeichnete ein dem Böhme'schen nicht unähnliches Sendungsbewusstsein aus, ihr „europäischer Geist“ glich einer Ideologie und Teilen der Gemeinschaft diente sie wohl auch als Identifikationsfigur. Als die Differenzen überhand zu nehmen drohten, wurde auf der Hauptver-

23 Was glauben Sie eigentlich? Hg. von der Deutschen Unitarier Religionsgemeinschaft e.V. Hamburg/Ravensburg 2000, S. 19.

${ }^{24}$ Kahl: Strömungen. 
sammlung 1985 überraschend vorgeschlagen, sie zur Ehrenpräsidentin zu ernennen.

„Die interessierte Öffentlichkeit nahm ,Europas andere Religion“ enthusiastisch auf", stellte Hunke zufrieden in ihrer Ansprache zur Verleihung der Ehrenpräsidentschaft fest, doch ihr war auch bewusst, wie umstritten ihr Führungsanspruch innerhalb der Unitarier war, wenn sie formulierte: „... Und die Unitarier? Einzelne Kreise in der Religionsgemeinschaft waren hingerissen ... Andere schwiegen lange ... Bei einigen rief es von vornherein Frustration hervor: Im Zuge einer alles ergreifenden Demokratisierung schien hier Autorität zu drohen, ein Dogma, die Freiheit zu beschneiden ..." 25

Die von Hunke geleitete Arbeitsgemeinschaft Europas eigene Religion war kein legitimiertes Gremium, wollte jedoch eigene Leitgedanken durchsetzen, die aber in wesentlichen Punkten dem unitarischen Selbstverständnis widersprachen. Fast vier Jahre dauerte das Ringen der Ehrenpräsidentin um die Deutungshoheit innerhalb der Religionsgemeinschaft, bevor sie feststellte: „Dies ist nicht mehr die Gemeinschaft, in die ich eingetreten bin." In den Monaten nach ihrem Austritt taten es ihr etliche Mitglieder gleich, mehrere Gemeinden traten geschlossen aus und gründeten 1989 den Bund deutscher Unitarier, Religionsgemeinschaft europäischen Geistes.

\section{Angriffe von Links}

Im selben Jahr begann eine Welle der Verleumdung über der Religionsgemeinschaft zusammenzuschlagen. Sie sei eine „völkisch-rassistische Sekte“ und „,nazistische Tarnorganisation“ lautete der Vorwurf, der aus linken Kreisen erhoben wurde. Böhme und Hunke dienten dabei als Belege. Der Versuch scheiterte, diese Anwürfe gerichtlich verbieten zu lassen, da es sich um eine „subjektive Einschätzung“ handele, die dem Wahrheitsbeweis nicht zugänglich sei. Der Schaden für die Gemeinschaft, ihre öffentliche Reputation und die Funktionsträger war immens. Die Wirkung öffentlicher Erklärungen des Vorstandes blieb vergleichsweise gering. Noch Ende der 1990er Jahre gab es parlamentarische Anfragen linker Politiker zu den Beziehungen der Deutschen Unitarier in das rechtsextreme Spektrum und Antifa-Demonstrationen bei Unitariertagen.

„Da wir keine anderen Prinzipien anwenden als die demokratischen, sind Trennungen nicht per Dekret zu verfügen, sondern nur argumentativ durchsetzbar. Dies ist einerseits eine Stärke, führt aber andererseits dazu, dass wir durch Demagogen angreifbar werden“, beschrieb 1991 der scheidende Präsident Horst Prem das Dilemma der Gemeinschaft, aber - so weiter - dies „sollte uns nicht davon abbringen, unser Toleranzprinzip weiter in argumentativer Form zu praktizieren. In diesem Sinne wünsche ich unserer Religionsgemeinschaft und jedem

\footnotetext{
25 Sigrid Hunke: Und aus Einem alles - und in allem Eins: Ansprache bei der Verleihung der Ehrenpräsidentschaft der Deutschen Unitarier. In: unitarische blätter. Bd. 4. Juli/August 1985, S. 156-157, hier S. 157.
} 
einzelnen die Kraft und Ausdauer, diesen konsequenten Weg auch in Zukunft zu gehen. Er wird sich auf Dauer lohnen. '"26

\section{4 Öffnung und Aufbruch}

Die 2011 auf der Hauptversammlung verabschiedete Kasseler Erklärung 27 sollte keinen Zweifel an der Lauterkeit der deutschen Unitarier mehr lassen. Wir stehen fest zu den unitarischen Prinzipien von Freiheit, Vernunft und Toleranz, wie schon Walbaum sie formuliert hat, und zu der von Christian Elßner 1882 in Alzey formulierten, ,protestantischen Mission, der Fort- und Weiterentwicklung religiöser Erkenntnis“ und der Pflicht, ,alles entschieden zurückzuweisen, was dem Menschengeiste zu hemmenden Fesseln werden könnte“.28

Die Angriffe von außen ließen die Gemeinschaft sich verstärkt nach innen orientieren. Die inhaltliche Arbeit überwog, die Öffentlichkeitsarbeit trat in den Hintergrund. Nach den ersten Grundgedanken von 1977, die die Leitgedanken von 1957 abgelöst hatten, wurde 1995 auf der Hauptversammlung in Hitzacker eine weiterentwickelte Fassung verabschiedet. Es scheint als sei alle zwanzig Jahre eine neue Generation der religiösen Grundaussagen erforderlich.

Apropos Generationenwechsel: Verjüngte sich der Vorstand Mitte der 1970er Jahre erstmals dramatisch, begann 1993 die ,feminine Periode': sechs von neun Vorstandsmitgliedern waren weiblich - darin eingeschlossen die Präsidentin und die Vizepräsidentin. Auf die erste deutsche Bundeskanzlerin mussten wir da noch weitere zwölf Jahre warten! In der Zwischenzeit prägten Diskussionen um die Rolle von Ritualen und Spiritualität die inhaltlichen Auseinandersetzungen, auch der Begriff „Religionsgemeinschaft“ und das Symbol werden seit langem kontrovers debattiert. Mit dem im Eigenverlag erschienenen Buch $W$ as glauben Sie eigentlich?29 beginnt ab 2001 wieder eine verstärkte Orientierung nach außen.

2009 fiel der Startschuss für das auf drei Jahre angelegte „Projekt 2000+“, für das die Mitglieder über die üblichen Beiträge hinaus beträchtliche Mittel gespendet hatten. Das Ziel war es, die Erfahrungen der amerikanischen unitarischen Freunde zu nutzen, um die Religionsgemeinschaft nach innen zu stärken, eine Öffnung nach außen zu fördern und neue Interessenten für die unitarische Idee zu gewinnen. Auch wenn nicht alle Ziele erreicht wurden, so gab das Projekt eine Vielzahl von Impulsen in die Gemeinschaft. Auf der Hauptversammlung 2015 in Worms wurde dann auch beschlossen, den Namen der Gemeinschaft in Unitarier - Religionsgemeinschaft freien Glaubens zu ändern.

\footnotetext{
${ }^{26}$ Horst Prem: Aus der Gemeinschaft - Brief des zurückgetretenen Präsidenten. In: unitarische blätter. Bd. 3. Mai/Juni 1991, S. 136-137, hier S. 137.

27 http://www.unitarier.de/unitarier/wer-wir-sind/geschichte/kasseler-erklaerung/.

${ }^{28}$ Elßner: Materialien zum Religionsunterricht, S. 88.

${ }^{29}$ Was glauben Sie eigentlich? Hg. von der Deutschen Unitarier Religionsgemeinschaft e.V. Hamburg/Ravensburg 2000.
} 